\title{
Exploration of high-fidelity simulation: Nurse educators' perceptions and experiences at a school of nursing in a resource-limited setting
}

\author{
T Munangatire, ${ }^{1}$ BScNS, MPhil (HSE); N Naidoo, ${ }^{2}$ MMedSci, MEd \\ ${ }^{1}$ Paray School of Nursing, Thaba Tseka, Lesotho \\ ${ }^{2}$ Division of Physiotherapy, Department of Health and Rehabilitation Sciences, Faculty of Health Sciences, University of Cape Town, South Africa
}

Corresponding author: T Munangatire (takamunangatire@gmail.com)

Background. Simulations are defined as situations where models are used for practice and to gain experience that will enhance students' practical skills. The use of simulations in clinical skills training can stimulate deep learning and help students to bridge the gap between theory and practice in nursing. This has been revealed in many studies where simulations positively impacted on clinical decision-making and patient care, and there has been great interest in the use of simulation in nurse training. However, the introduction of technologically driven simulators, especially in resourceconstrained settings, has been met with mixed feelings.

Objective. To explore the perceptions and experiences of nurse educators in using high-fidelity simulation (HFS) in teaching.

Methods. A qualitative case study design was utilised. Seven educators at a school of nursing, which has HFS, participated in a focus group discussion. Data were thematically analysed.

Results. Four themes emerged from the educators' experiences and perceptions. The use and benefits of HFS were generally accepted by educators. They valued its positive impact on learning outcomes in learners and the ability to simulate more complex scenarios during training. Lack of prior planning, inadequate training and lack of resources impacted negatively on the effective use and implementation of HFS.

Conclusion. The results indicated that nurse educators perceived HFS as a learning pedagogy that can improve students' learning outcomes if used effectively. They believed that to realise the potential of HFS, more support should be provided through training, the availability of necessary resources, and improved planning and organisation.

Afr J Health Professions Educ 2017;9(1):44-47. DOI:10.7196/AJHPE.2017.v9i1.739

Simulation is the art and science of recreating a clinical scenario in an artificial setting to allow for deliberate teaching and learning of clinical skills. ${ }^{[1]}$ Examples of simulation include the use of standardised patients, models and mannequins, which may be low, medium or high fidelity. A high-fidelity simulator, such as a state-of-the-art mannequin, can be programmed to produce physiological functions, such as palpable pulses, voices and abdominal sounds through computer interfaces. ${ }^{[2]}$ Changes in training of nurses and the progressive culture of teaching and learning support the use of simulation to maximise and enhance clinical skills training. ${ }^{[2]}$ High-fidelity simulation (HFS) uses advanced technology to produce most human physiological responses in a mannequin, while medium-fidelity simulation provides only basic human responsive functions, with low-fidelity simulation mannequins being static and non-responsive. ${ }^{[2]}$

While there has been an explosion of research on HFS in the developed world, there are relatively few studies emerging from the developing world..$^{[3]}$ Current evidence shows that the use of HFS in nurse training institutions has been met with mixed reactions, which has impacted on the use of HFS at these institutions ${ }^{[4]}$ In cases where HFS has been well received and effectively used, it has resulted in improved learning of clinical skills. ${ }^{[5]}$ It therefore stands to reason that an institution that introduces HFS will benefit from establishing its acceptability among educators. The benefits of knowing how educators perceive the value of HFS will give an indication of the likelihood of it being used in teaching and training.
Against a background of slowly improving health outcomes, Lesotho acquired HFS with the hope of improving the quality of nursing skills training. HFS equipment was distributed to six nursing schools in Lesotho. For HFS to facilitate improved clinical skills learning outcomes in these institutions, it has to be accepted and effectively used by nurse educators and students. This study was conducted to explore nurse educators' perceptions and experiences of using HFS in learning clinical skills in one of the resource-constrained schools of nursing.

\section{Literature review HFS as a learning pedagogy}

As a pedagogical strategy, HFS is supported by learning theories, including constructivism, experiential learning and situated cognition. When making use of HFS during learning sessions, students practise skills by means of clinical scenarios, where they construct meaning out of these scenarios through deliberate practice. Students need to understand that the practice that takes place in the clinical skills laboratory later needs to be transferred to the clinical area. Therefore, theories suggest that HFS as a teaching and learning strategy shifts learning from a predominantly behaviourist pedagogy (teacher centred) towards more student-centred approaches. ${ }^{[2]}$ The student-centred approaches supported by HFS include experiential learning and situated cognition, where HFS allows students to make mistakes in the simulation and then transform and apply the learned experiences to tasks, 
interactions and cultural dynamics in different situations and contexts, such as various departments of the clinical area. ${ }^{[7,8]}$

\section{Theoretical framework}

The National League of Nursing Framework (NLNF), developed by Jeffries, ${ }^{[1]}$ is important in understanding the concepts that influence the acceptability of HFS. ${ }^{[9]}$ This framework suggests that effective HFS use depends on several factors (Fig. 1). Jeffries ${ }^{[1]}$ acknowledges the role of the teacher as a designer, supervisor and implementer of simulation in nurse education. To ensure successful use of simulation, the teacher must have a good perception of simulation, be comfortable using the technology, and be a good facilitator and evaluator of the learning process. ${ }^{[1]}$ The NLNF has been endorsed and utilised in evaluating simulation use by several researchers ahead of other models, such as the Expert Performance Approach. ${ }^{[1,10]}$ Therefore, the Jeffries ${ }^{[1]}$ model remains the most relevant and was used to guide this study.

\section{Constructs shaping experiences and perceptions}

Using HFS in teaching presents educators with mixed experiences - some exciting and some frustrating. The experiences are shaped by constructs, such as planning for simulation, training on how to use simulation, the availability of resources and motivation for using simulation. ${ }^{[4]}$ Educators' experiences of using HFS influence their perceptions and adoption of HFS in teaching. ${ }^{[4]}$ Adoption of HFS as pedagogy for clinical skills without carefully planning for its use creates a negative perception among educators. ${ }^{[10,11]}$ Issenberg et al. ${ }^{[10]}$ contend that HFS is complex, and for effective use it requires excellent planning and organisational contextualisation. Furthermore, lack of time, support, appropriate equipment and fear of using HFS simulation as a pedagogy create negative perceptions, and result in underutilisation. ${ }^{[12]}$ Findings to date suggest that there are mixed perceptions among lecturers regarding the impact on training of using HFS in teaching. ${ }^{[10]}$ In Dowie and Phillips ${ }^{\text {[13] }}$ study, $90 \%$ of educators were using HFS, although only $35 \%$ of them had been trained in its use. However, Schlairet's ${ }^{[14]}$ findings revealed that only $50 \%$ of trained educators were using HFS in teaching. This suggests that motivation and the level of support from colleagues and administrative staff influence educators' use of HFS rather than training alone. ${ }^{[1]]}$

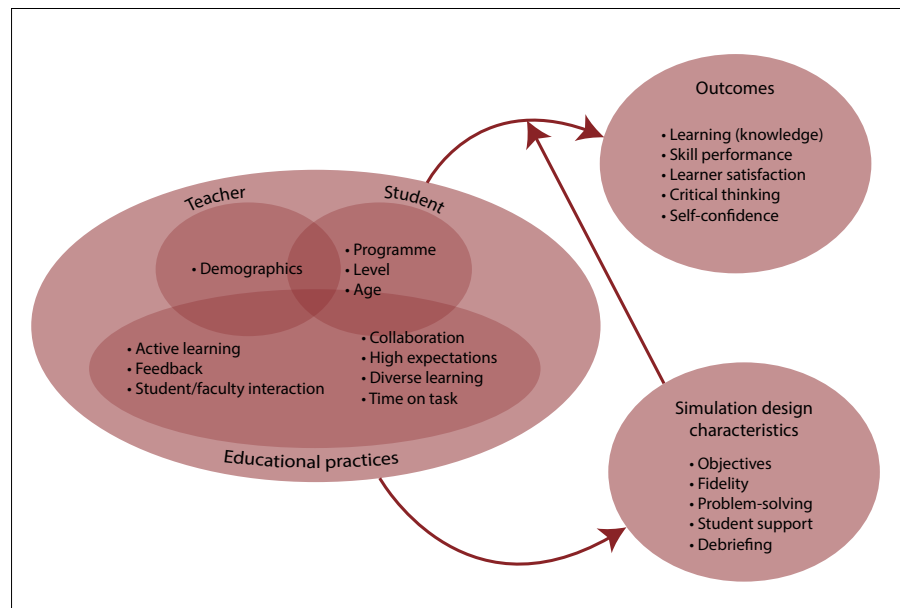

Fig. 1. National League of Nursing Framework. ${ }^{[1]}$
In summary, this study explored the perceptions and experiences of nurse educators' use of HFS in teaching, as its acceptability by educators as a teaching strategy is important for effective use.

\section{Methods \\ Research design}

A descriptive, qualitative case study design was utilised to explore nursing educators' perceptions and experiences in a focus group discussion.

\section{Population and sample}

All 10 educators who teach at our nursing school were identified as the population for this case study. From this population, a sample of seven educators was purposively selected to participate in the study, based on the inclusion criteria stated above. These seven educators were selected because they were using HFS in their teaching.

\section{Data collection}

Data were collected during a once-off focus group discussion, which was guided by an interview schedule adapted from Krueger and Caseyss ${ }^{[15]}$ guidelines on focus group interviews. Adaptations to the guidelines were made based on literature and the opinions of two researchers who validated the semi-structured interview guide. The researcher and the assistant prepared the venues and seating arrangement in a semicircle to promote interaction.

The researcher moderated the focus group discussion, while the assistant managed digital voice recorders and took notes. Anonymity was ensured by identifying participants by number rather than name. The focus group discussion lasted for 55 minutes.

To ensure the credibility of the study, the methods were well described, and the data were recorded and transcribed verbatim by the researcher. The co-researcher and participants checked the transcription for accuracy.

\section{Data analysis}

The data recordings were transcribed by the researcher, who organised the data into paper records for ease of thematic analysis. Thematic analysis was suitable for this descriptive qualitative study because it is not closely tied to any theory of qualitative research, and allows for flexibility of analysis, resulting in the detailed description of data.

Data analysis was an iterative and reflexive process to ensure richness of the analysis. It commenced with the coding process, which involved recognising (seeing) an important moment and encoding (seeing it as something) it prior to a process of interpretation. The encoding organised the data in such a way that themes could be identified and developed. The encoding process resulted in the development of a codebook, which served as a data management tool for organising segments of similar or related text to assist in interpretation. As a way of testing the reliability of the codes, the researcher invited the co-researcher to also code the transcripts; the co-researcher's codes were found to be similar to those of the researcher.

\section{Results}

This study explored nursing educators' experiences and perceptions of using HFS in teaching. Participants had positive and negative experiences and perceptions of HFS, which are described under the themes that emerged from the focus group discussion data: planning, training, resources, benefits and limitations (Table 1). 


\section{Discussion}

This study explored nurse educators' perceptions and experiences of HFS in teaching to establish how acceptable HFS would be in a resource-limited setting.

Participants perceived HFS to be an important teaching strategy, but pointed out that effective implementation requires careful planning. Hyland and Hawkins ${ }^{[9]}$ and Adamson ${ }^{[1]}$ noted that lack of adequate preparation for the introduction of HFS results in non-use. However, in this study, lack of planning did not impede the use of HFS in teaching clinical skills

Lack of training did not discourage the use of HFS for teaching, as educators were highly motivated and willing to teach using HFS. They appreciated the benefits of being able to simulate rare scenarios, teaching problem-solving skills and improving student confidence. ${ }^{[4]}$ There is a need to support this motivation and willingness through formally training educators, which can potentially promote HFS use and destigmatise the educators' experiences and perceptions of not using or improperly using HFS. ${ }^{[5,7,13]}$ Training will capacitate educators to use HFS strategically and to maximise its benefits in teaching. ${ }^{[6,12]}$ Despite challenging experiences due to lack of formal training, educators used HFS because they believed it to be a worthwhile investment that could improve student learning outcomes in resource-limited settings.

HFSs are some of the resources required in teaching clinical skills in nursing, but cannot be used in isolation. ${ }^{[12]}$ Additional resources, such as time and appropriate equipment, are needed to facilitate effective teaching using HFS, otherwise educators become frustrated and discouraged from using it. ${ }^{[13]}$ The need for these resources, together with technological support in the simulation room, was clearly verbalised in our study, confirming the need to holistically plan and procure all resources necessary to effectively operationalise HFS in teaching. In this way, nurse educators will have satisfying experiences and consequently readily accept the use of HFS in teaching.

Table 1. Findings

Theme Descriptors Quote

Planning Some participants were not pleased with the implementation of HFS at the school and felt that the resource had been provided without giving due recognition to other priority needs. Some people were frustrated that HFS had been introduced without consultation. They explained that the frustration was evident in the low use of HFS as a teaching resource

Training

Resources

Benefits

Limitations lacked adequate training and were not able to fully use HFS with the initial level of training

The participants perceived HFS use as a demanding pedagogy, requiring technical know-how, careful planning, time, teamwork, and innovation in creating simulation scenarios for effective implementation and type. Additional resources were needed, such as mannequins, part-task trainers, technical support, monitoring cameras and dedicated space for projection, where students may observe demonstrations without being present in the simulation rooms

Participants were strongly in favour of the use of HFS because of students' HFS promoted skills transfer from the lecturer to the students

Some reported that HFS allows simulation of uncommon procedures, facilitating traditionally difficult-to-teach skills, such as problem-solving and critical thinking

Participants felt that the ability to transfer skills from the simulation to the
'It is an excellent teaching device but it is not meeting what we want at that point in time.' (Educator 3)

'... they suddenly just bring those things [HFS] to us.' (Educator 3)

'Because if really our institutions cried for those simulators, we couldn't be facing challenges in terms of utilising them.' (Educator 1)

'When we were first oriented on how to use high fidelity simulation, I was so excited and I will believe that I will be able to use it and it will enhance, ahh my teaching ... ? (Educator 6)

Participants emphasised that there was insufficient equipment, both in quantity positive learning outcomes and improved confidence, and because they felt that clinical area may not be easy

Some participants noted that there were limitations to the level of fidelity or the extent to which HFS resembles a real human being. HFS was, for example, able to imitate a human being physically, but failed to do so psychologically
'... I might not have the time for preparation and planning because I might have to feed the doll information ... ? (Educator 2)

'My recommendation is as I mention the issue of technical somebody like in a school lab ... .'

(Educator 5)

'... high fidelity wouldn't be part of our high priority needs.' (Educator 4)

‘... simulators help build up the confidence and polish up the skill ....' (Educator 3)

'... simulator helps in the transfer of skill from the instructor to the students.' (Educator 3)

'... the high fidelity ones are more effective in other clinical situations that are rare ... ? (Educator 1)

'... that thing is not a person, when the student is practising on that thing it might be easy.' (Educator 3)

‘... they are more effective on the skills part, but the attitudes, nurse-patient relationship, it's not very effective ... ? (Educator 5)

'It's like you cannot do everything on high fidelity, there is a limited number, that limitation frustrated me.' (Educator 5) 
Previous studies have revealed that educators view HFS as an effective teaching strategy, but noted that it has a number of limitations. ${ }^{[13]}$ This resonates well with the findings in our study, where educators perceive HFS as a beneficial teaching strategy, even though it has inherent limitations. Our study found that educators believe that HFS improves learning outcomes in simulation, although the nurse educators thought that it is difficult to transfer these skills to actual practice. Young and Shellenbarger' ${ }^{36]}$ findings align with these findings, which showed that nurse educators' use of HFS depends on whether or not they are comfortable using the technology and believe that HFS promotes learning and improves learning outcomes. However, its use is challenging owing to the complexities associated with working with simulation equipment.

Nurse educators view HFS as a worthwhile strategy for use in teaching, and the reported benefits of its use act as a motivation for them to adopt it. This suggests that nurse educators are in favour of using HFS in teaching clinical skills.

Poor utilisation of HFS by educators may stem from negative experiences surrounding the use of simulation. Poor planning in introducing HFS, lack of resources to support its use, inadequate training, and the limitations of HFS in teaching result in negative experiences and hence poor utilisation by educators. To have HFS accepted and used effectively, it is essential to create adequate implementation plans, acquire the necessary additional resources, and provide comprehensive training for educators.

\section{Conclusion}

HFS is perceived as a valuable teaching strategy that improves students' competence, motivating educators to use HFS in teaching clinical skills. However, if it is not properly implemented, educators may experience frustration and disappointment, with the result that the benefits of HFS may be diminished, leading to under-utilisation or non-use. Therefore, setting up HFS and implementing it should be carefully planned, and educators should be formally trained and given technical support and any additional resources needed for teaching students to use HFS. These measures will increase the adoption of HFS which, in turn, may improve the training of nurses.

\footnotetext{
1. Jeffries PR. A framework for designing, implementing, and evaluating simulations used as teaching strategies in nursing. Nurs Educ Perspect 2005;26(2):96-103

2. Bux A. Nurses' perceptions of the usefulness of high fidelity simulation technology in a clinical education program. ProQuest 2009;503.

3. Davies J, Alinier G. The growing trend of simulation as a form of clinical education: A global perspective. Int Para Prac 2011:1(2):58-62. http://dx.doi.org/10.12968/ippr.2011.1.2.58

4. Reid-Searl K, Happell B, Vieth L, et al. High fidelity patient silicone simulation: A qualitative evaluation of nursing students' experiences. Collegian 2012;19(2):77-83.

5. Cooper S, Cant R, Porter J, et al. Simulation based learning in midwifery education: A systematic review. Women Birth 2012;25(2):64-78. http://dx.doi.org/10.1016/j.wombi.2011.03.004

6. Young $\mathrm{PK}$ Shell: J Nurs Educ 2012;51(8):422-428. http://dx.doi.org/10.3928/01484834-20120523-02

7. Paige JB, Daley BJO. Situated cognition: A learning framework to support and guide high-fidelity simulation. Clin Simul Nurs 2009;5(3):e97-e103. http://dx.doi.org/10.1016/j.ecns.2009.03.120

8. Lea St, Stephenson D, Troy J. Higher education students' attitudes to student-centred learning. Beyond 'educational bulimia'? Studies Higher Educ 2003;28(3):321-334. http://dx.doi.org/10.1080/03075070309293

9. Hyland JR, Hawkins MC. High-fidelity human simulation in nursing education: A review of literature and guide for implementation. Teach Learn Nurs 2009;4(1):14-21. http://dx.doi.org/10.1016/j.teln.2008.07.004

10. Issenberg BS, McGaghie WC, Petrusa $\mathrm{E}$ et al. Features and uses of high-fidelity medical simulations that lead to effective learning. A BEME systematic review. Med Teach 2005;27(1):10-28. http://dx.doi.org 10.1080/01421590500046924

11. Adamson K. Integrating human patient simulation into associate degree nursing curricula: Faculty experiences barriers, and facilitators. Clin Simul Nurs 2010;6(3):e75-e81. http://dx.doi.org/10.1016/.j.ecns.2009.06.002

12. Garrett B, MacPhee M, Jackson C. High-fidelity patient simulation: Considerations for effective learning. Nurs Educ Perspect 2010;31(5):309-313.

13. Dowie I, Phillips C. Supporting the lecturer to deliver high-fidelity simulation. Nurs Standard 1987;25(49):35-40. http:// dx.doi.org/10.7748/ns2011.08.25.49.35.c8651

14. Schlairet MC. Simulation in an undergraduate nursing curriculum: Implementation and impact evaluation. J Nurs Educ 2011;50(10):561-568. http://dx.doi.org/10.3928/01484834-20110630-04

15. Krueger RA, Casey MA. Designing and conducting focus group interviews. Social Anal Select Tools Techn 2002;4(23):4-24.
} 